\title{
ON THE MODULAR FUNCTIONS ARISING FROM THE THETA CONSTANTS
}

\author{
UĞUR S. KIRMACI
}

\begin{abstract}
Some modular functions arising from the theta constants $\vartheta_{2}(\tau), \vartheta_{3}(\tau), \vartheta_{4}(\tau)$ are investigated. Let $n$ be an odd square-free positive integer as in [4,7]. It is obtained a necessary and sufficient condition that $\varphi_{\delta, \rho, 3}(\tau)=\prod_{\delta|n, \rho| n}\left(\frac{\vartheta_{3}(\delta \tau)}{\vartheta_{3}(\rho \tau)}\right)^{r_{\delta}}$ is invariant with respect to transformations in $\theta(n)$. Also, It is deduced that $\varphi_{\delta, \rho, i}(\tau)$ is a modular function on $P^{-2} \theta(n) P^{2}$, $\theta(n), P^{-1} \theta(n) P$, for $i=2,3,4$, respectively. Thus, the result of L. Wilson's paper [7] is generalized. Furthermore, let $m$ and $n$ denote positive integers. Let $r, r_{1}, r_{2}$ be integers such that $r(m-1)(n+1) \equiv 0(\bmod 8), r_{1}(m-1)(n-1) \equiv 0(\bmod 8), r_{2}^{2}(n-m)(n m-1) \equiv 0(\bmod 8)$, it is shown that $T_{m, n, i}^{r}(\tau)=\left(\frac{\vartheta_{i}(\tau) \vartheta_{i}(n \tau)}{\vartheta_{i}(m \tau) \vartheta_{i}(m n \tau)}\right)^{r}, H_{m, n, i}^{r_{1}}(\tau)=\left(\frac{\vartheta_{i}(m \tau) \vartheta_{i}(n \tau)}{\vartheta_{i}(\tau) \vartheta_{i}(m n \tau)}\right)^{r_{1}}$ and $\Phi_{m, n, i}^{r_{2}}(\tau)=\left(\frac{\vartheta_{i}(m \tau)}{\vartheta_{i}(n \tau)}\right)^{r_{2}}$ are modular functions on $\theta(m n)$, when $i=3$. Similar results are deduced for $P^{-2} \theta(m n) P^{2}$ and $P^{-1} \theta(m n) P$, the suffixes 3 being replaced by 2 and 4 , respectively. Therefore, the modular functions used in B. C. Berndt's paper [1] is rewritten for theta constants.
\end{abstract}

\section{Introduction}

We shall use $\chi$ to denote the upper half-plane, $Z$ for the set of rational integers and $\Gamma(1)$ for the modular group. Let be $U=\left(\begin{array}{ll}1 & 1 \\ 0 & 1\end{array}\right), V=\left(\begin{array}{cc}0 & -1 \\ 1 & 0\end{array}\right), W=\left(\begin{array}{ll}1 & 0 \\ 1 & 1\end{array}\right)$, $P=\left(\begin{array}{rr}0 & -1 \\ 1 & 1\end{array}\right)$.

$\Gamma_{u}(2), \Gamma_{v}(2)$ and $\Gamma_{w}(2)$ are defined by

$$
\begin{aligned}
& \Gamma_{u}(2)=\{S \in \Gamma(1): S \equiv I \text { or } S \equiv U(\bmod 2)\} \\
& \theta=\Gamma_{v}(2)=\{S \in \Gamma(1): S \equiv I \text { or } S \equiv V(\bmod 2)\} \\
& \Gamma_{W}(2)=\{S \in \Gamma(1): S \equiv I \text { or } S \equiv W(\bmod 2)\}
\end{aligned}
$$

where $I$ is the unit matrix. The three subgroups $\Gamma_{u}(2), \Gamma_{v}(2)$ and $\Gamma_{w}(2)$ are conjugate [6].

Received January 21, 2002; revised April 22, 2002.

Key words and phrases. Theta constants, modular functions. 
The subgroup $\theta=\Gamma_{v}(2)$ of $\Gamma(1)$ is generated by $U^{2}$ and $V$. For an odd positive integer $n$, the set of elements in $\theta$ of the form $\left(\begin{array}{ll}a & b \\ c n & d\end{array}\right)$ is a subgroup of $\theta$ which will be denoted $\theta(n)$. The subgroup $\Gamma_{0}(k)$ is defined to be the set of elements in $\Gamma(1)$ of the form $\left(\begin{array}{ll}a & b \\ c k & d\end{array}\right)$, where $k$ is a positive integer. $\Gamma_{0}(2 n)$ and $\theta(n)$ are conjugate subgroups of $\Gamma(1)$. That is, $\Gamma_{0}(2 n)=W^{-n} \theta(n) W^{n}$. [7]

Let $F$ denote the Ford fundamental region of $\theta(n)$, where $n$ denotes an odd squarefree positive integer. A complete set of non-equivalent parabolic points of $\theta(n)$ in $\bar{F}$ is given by

$$
0, i \infty \text { and } P(n)=\{2 / \Delta: \Delta \mid n, \Delta>0\} \cup\{1 / \Delta: \Delta \mid n, \Delta>0\}
$$

[4], [7, Theorem 1].

We recall the theta constants $\vartheta_{2}(\tau), \vartheta_{3}(\tau)$ and $\vartheta_{4}(\tau)$ defined by

$$
\vartheta_{2}(\tau)=\sum_{n \in Z} q^{\left(n+\frac{1}{2}\right)^{2}}, \vartheta(\tau)=\vartheta_{3}(\tau)=\sum_{n \in Z} q^{n^{2}}, \vartheta_{4}(\tau)=\sum_{n \in Z}(-1)^{n} q^{n^{2}}
$$

for $\tau \in \chi$ and $q=e^{\pi i \tau}$. The Dedekind Eta function

$$
\eta(\tau)=e^{\pi i \tau / 12} \prod_{n=1}^{\infty}\left(1-e^{2 \pi i n \tau}\right)
$$

is a cusp form of weight $1 / 2$ on $\Gamma(1)$ and satisfies

$$
\eta(M \tau)=v_{\eta}(M)(c \tau+d)^{1 / 2} \eta(\tau)
$$

for all $M=\left[\begin{array}{ll}a & b \\ c & d\end{array}\right] \in \Gamma(1)$. An important connection between $\vartheta(\tau)$ and $\eta(\tau)$ is given by.

$$
\vartheta(\tau)=\eta^{2}\left(\frac{\tau+1}{2}\right) / \eta(\tau+1)
$$

$[3]$.

Let $\Gamma$ be a subgroup of $\Gamma(1)$. If $f(\tau)$ is a modular form of weight $k$ for $\Gamma$ with multiplier system $v$, we write $f(\tau) \in M(\Gamma, k, v)$. If $f \in M(\Gamma, k, v)$ and $L \in \Gamma(1)$, the $L$-transform $f_{L}$ of $f$ is defined by

$$
f_{L}(\tau)=f(\tau) \mid L=\{\mu(L, \tau)\}^{-1} f(L \tau)
$$

Here, $\mu(L, \tau)=(c \tau+d)^{k}$, for $L=\left(\begin{array}{ll}* & * \\ c & d\end{array}\right)$. If $f_{1} \in M\left(\Gamma, k_{1}, v_{1}\right)$ and $f_{2} \in M\left(\Gamma, k_{2}, v_{2}\right)$, then $f_{1} . f_{2} \in M\left(\Gamma, k_{1}+k_{2}, v_{1} . v_{2}\right)$ and $f_{1} / f_{2} \in M\left(\Gamma, k_{1}-k_{2}, v_{1} / v_{2}\right)$. 
Lemma 1. Suppose that $f \in M(\Gamma, k, v)$ and $L \in \Gamma(1)$. Then we have $f_{L} \in$ $M\left(L^{-1} \Gamma L, k, v^{L}\right)$. [6]

Lemma 2. The functions $\vartheta_{2}(\tau), \vartheta_{3}(\tau)$ and $\vartheta_{4}(\tau)$ are entire modular forms of weight $1 / 2$ for the groups $\Gamma_{u}(2), \Gamma_{v}(2)$ and $\Gamma_{w}(2)$, respectively. Further,

$$
\begin{aligned}
\vartheta_{3}(\tau) \mid P & =e^{-\frac{1}{4} \pi i} \vartheta_{4}(\tau) \\
\vartheta_{3}(\tau) \mid P^{2} & =e^{-\frac{1}{4} \pi i} \vartheta_{2}(\tau)
\end{aligned}
$$

Also, for $n \geq 0, \vartheta_{2}^{n}, \vartheta_{3}^{n}$ and $\vartheta_{4}^{n}$ are entire modular forms of weight $n / 2$ for the groups $\Gamma_{u}(2), \Gamma_{v}(2)$ and $\Gamma_{w}(2)$, respectively $[6]$.

Lemma 3. Let $n$ be an odd square-free positive integer. For each divisor $\delta$ of $n$, with $\delta>1$, let $r_{\delta}$ be any integer and let $r_{1}=0$. Then

$$
f(\tau)=\prod_{\delta \mid n}\{\vartheta(\delta \tau) / \vartheta(\tau)\}^{r_{\delta}}
$$

is invariant with respect to the transformations in $\theta(n)$ if and only if the following conditions hold:

(i) $\sum_{\delta \mid n}(\delta-1) r_{\delta} \equiv 0(\bmod 8)$

(ii) $\prod_{\delta \mid n} \delta^{r_{\delta}}$ is the square of a rational number

The set of functions given by (3) and satisfying (i) and (ii) will be denoted $F(n)$. The functions in $F(n)$ are modular functions on $\theta(n)$, where $\vartheta(\tau)=\vartheta_{3}(\tau)$ [7, Theorem 3].

For relatively prime integers $x$ and $y$ with $x \neq 0$ and $y$ odd, define

$$
\left(\frac{x}{y}\right)^{*}=\left(\frac{x}{|y|}\right) \quad \text { and } \quad\left(\frac{x}{y}\right)_{*}=\left(\frac{x}{|y|}\right)(-1)^{\frac{1}{4} \varepsilon(x) \varepsilon(y)}
$$

where $\left(\frac{x}{|y|}\right)$ is the Jacobi symbol and $\varepsilon(x)=x /|x|$. We also define $\left(\frac{0}{ \pm 1}\right)^{*}=\left(\frac{0}{1}\right)_{*}=1$ and $\left(\frac{0}{-1}\right)_{*}=-1$.

The multiplier system for $\vartheta_{3}(\tau)$ is given by

$$
v(A)= \begin{cases}\left(\frac{d}{c}\right)^{*} \exp \left(\frac{1}{4} \pi i c\right) & \text { if } A \equiv V(\bmod 2) \\ \left(\frac{c}{d}\right)_{*} \exp \left(\frac{1}{4} \pi i(d-1)\right) & \text { if } A \equiv I(\bmod 2)\end{cases}
$$

where $A=\left(\begin{array}{ll}* & * \\ c & d\end{array}\right) \in \theta$. Thus, $v(A)$ is an $8^{\text {th }}$ root of unity and

$$
\vartheta(A \tau)=v(A)(c \tau+d)^{1 / 2} \vartheta(\tau), \quad \tau \in \chi, \quad[7]
$$

This paper is a continuation of previous work [2]. We will develop some results in [1], [4], [5] and [7]. The valences of functions in this paper are the same as those constructed 
in [4] and [7]. Certain theorems given in [4] and [7] carry over to the present setting with only minor alterations in their proofs. The main results of this paper are the following theorems.

\section{The Modular Functions $\varphi_{\delta, \rho, i}(\tau)$}

Now, we define the functions $\varphi_{\delta, \rho, i}$ as follows:

$$
\varphi_{\delta, \rho, i}(\tau)=\prod_{\delta|n, \rho| n}\left\{\vartheta_{i}(\delta \tau) / \vartheta_{i}(\rho \tau)\right\}^{r}, \quad i=2,3,4
$$

Theorem 1. Let $n$ be an odd square-free positive integer. For each divisor $\delta$ and $\rho$ of $n$, with $\delta, \rho>1, \delta \neq \rho$, let $r=r_{\delta}$ be an integer and let $r_{1}=0$. Then $\varphi_{\delta, \rho, 3}$ is invariant with respect to the transformations in $\theta(n)$ if and only if the following conditions hold:

$$
\begin{aligned}
& \text { (i) } \sum_{\delta|n, \rho| n}(\rho-\delta) r_{\delta} \equiv 0 \quad(\bmod 8) \\
& \text { (ii) } \prod_{\delta|n, \rho| n}\left(\frac{n^{2}}{\delta \rho}\right)^{r} \text { is the square of a rational number. }
\end{aligned}
$$

Further $\varphi_{\delta, \rho, 3}$ is a modular function on $\theta(n)$.

Proof. It is enough to consider only those matrices $A=\left(\begin{array}{ll}a & b \\ n c & d\end{array}\right) \in \theta(n)$ such that $c \geq 0$ (since $A$ and $-A$ represent the same transformation) and such that $A \equiv V(\bmod 2)$ (since these generate $\theta(n))$. Let $B=\left(\begin{array}{ll}a & \delta b \\ \delta^{1} c & d\end{array}\right)$ and $C=\left(\begin{array}{ll}a & \rho b \\ \rho^{\prime} c & d\end{array}\right)$, where $\delta \delta^{\prime}=$ $\rho \rho^{\prime}=n$ so that $B, C \equiv V(\bmod 2)$ and $\delta A \tau=B \delta \tau, \rho A \tau=C \rho \tau$ Then using (4) and (5), we have

$$
\begin{aligned}
\varphi_{\delta, \rho, i}(A \tau) & =\prod_{\delta|n, \rho| n}\{\vartheta(\delta \tau) / \vartheta(\rho \tau)\}^{r_{\delta}} \\
& =\prod_{\delta|n, \rho| n}\{\nu(B) / \nu(C)\}^{r_{\delta}} \varphi_{\delta, \rho, i}(\tau) \\
& =\prod_{\delta|n, \rho| n}\left(\frac{d}{\delta^{\prime} \rho^{\prime}}\right)^{r_{\delta}} \cdot k \cdot \varphi_{\delta, \rho, i}(\tau)=\left(\frac{d}{\alpha}\right) \exp \left\{\frac{1}{4} \pi i c \beta\right\} \varphi_{\delta, \rho, i}(\tau)
\end{aligned}
$$

where, $k=\prod_{\delta|n, \rho| n}\left(\exp (1 / 4) \pi i c\left(\rho^{\prime}-\delta^{\prime}\right)\right) r_{\delta}, \alpha=\prod_{\delta|n, \rho| n}\left(\delta^{\prime} \rho^{\prime}\right)^{r_{\delta}}$ and $\beta=\sum_{\delta|n, \rho| n}\left(\rho^{\prime}-\right.$ $\left.\delta^{\prime}\right) r_{\delta}$

It remains to show that

(a) $\beta \equiv 0(\bmod 8)$ if and only if (i) holds, and

(b) $\left(\frac{d}{\alpha}\right)=1$ for all even integer $d$ relatively prime to $n$ if and only if (ii) holds. 
Since $n, \delta^{\prime}$ and $\rho^{\prime}$ are odd, $n^{2} \equiv\left(\delta^{\prime}\right)^{2} \equiv\left(\rho^{\prime}\right)^{2} \equiv 1(\bmod 8)$ and so $n \beta \equiv \sum_{\delta|n, \rho| n}(\rho-$ $\delta) r_{\delta}(\bmod 8)$ and $(\mathrm{a})$ follows. The "if" part of (b) is trivial. Assumming $\left(\frac{d}{\alpha}\right)=1$ for all even integers $d$ relatively prime to $n$ implies the same for all integers $d$ relatively prime to $n$. But if $\alpha$ is not a rational square, then by quadratic reciprocity and Dirichlet's Theorem there is a prime $p$ with $p \nmid n$ and $\left(\frac{p}{\alpha}\right)=-1$; so $\alpha$ must be a square, giving (ii).

By (1), we obtain

$$
\frac{\vartheta(\delta \tau)}{\vartheta(\rho \tau)}=\frac{\eta^{2}\left(\frac{\delta \tau+\delta}{2}\right) / \eta(\delta \tau+\delta)}{\eta^{2}\left(\frac{\rho \tau+\rho}{2}\right) / \eta(\rho \tau+\rho)}=\frac{\Phi^{2}\left(\tau^{\prime}\right) \Psi\left(2 \tau^{\prime}\right)}{\Phi\left(2 \tau^{\prime}\right) \Psi^{2}\left(\tau^{\prime}\right)}
$$

where $\tau^{\prime}=\frac{\tau+1}{2}, \Phi\left(\tau^{\prime}\right)=\frac{\eta\left(\delta \tau^{\prime}\right)}{\eta\left(\tau^{\prime}\right)}$ and $\Psi\left(\tau^{\prime}\right)=\frac{\eta\left(\rho \tau^{\prime}\right)}{\eta\left(\tau^{\prime}\right)}$.

Finally, we consider the expansions of $\varphi_{\delta, \rho, 3}(\tau)$ at the parabolic cusps $\infty, 0,2 / \Delta$ and $1 / \Delta$. We have

$\Phi(\tau)=\exp \left\{\frac{\pi i(\delta-1) r}{12} \tau\right\}\left(1+\sum_{k=1}^{\infty} a_{k} e^{2 \pi i k \tau}\right)$ and $\Psi(\tau)=\exp \left\{\frac{\pi i(\rho-1) r}{12} \tau\right\}\left(1+\sum_{k=1}^{\infty} b_{k} e^{2 \pi i k \tau}\right)$

as the Fourier expansions of $\Phi$ and $\psi$ at $\infty$. [3, p.103]. Hence, by $(6), \varphi_{\delta, \rho, 3}(\tau)$ has the Fourier expansion at $\infty$ of the form

$$
\varphi_{\delta, \rho, 3}(\tau)=1+\sum_{k=1}^{\infty} a_{k}^{\prime} e^{2 \pi i k \tau}
$$

We have

$$
\begin{aligned}
& \Phi(\tau)=\delta^{-r / 2} \exp \left\{\frac{\pi i(\delta-1) r}{12 \delta \tau}\right\}\left(1+\sum_{k=1}^{\infty} c_{k} e^{-2 \pi i k / \delta \tau}\right) \\
& \Psi(\tau)=\rho^{-r / 2} \exp \left\{\frac{\pi i(\rho-1) r}{12 \rho \tau}\right\}\left(1+\sum_{k=1}^{\infty} d_{k} e^{-2 \pi i k / \rho \tau}\right)
\end{aligned}
$$

as the Fourier expansion at $0[3, \mathrm{p} .103]$. Hence, by $(6), \varphi_{\delta, \rho, 3}(\tau)$ has the Fourier expansion at 0 of the form

$$
\varphi_{\delta, \rho, 3}(\tau)=\left(\frac{\rho}{\delta}\right)^{r / 2} \exp \left(\frac{\pi i r}{8 \tau}\left(\frac{\delta-\rho}{\delta \rho}\right)\right)\left(1+\sum_{k=1}^{\infty} \alpha_{k} e^{-\frac{2 \pi i k}{\tau} \gamma(\delta, \rho)}\right)
$$

where, $\gamma(\delta, \rho)$ is a rational function of $\delta$ and $\rho$. It is shown in [7] that $f(S \tau)$ has the form

$$
f(S \tau)=\sum_{k=0}^{\infty} a_{k} \exp \{2 \pi i k \tau /(2 n / \Delta)\}
$$


at the parabolic points $2 / \Delta$. Hence, we have

$$
\varphi_{\delta, \rho, 3}(S \tau)=\sum_{k=0}^{\infty} a_{k}^{\prime} \exp \{2 \pi i k \tau /(2 n / \Delta)\}
$$

where the $a_{k}^{\prime}$ are complex numbers independent of $\tau$ with $a_{0} \neq 0$ and $S=\left(\begin{array}{cc}2 & b \\ \Delta & d\end{array}\right)$. Replacing $\tau$ by $S^{-1} \tau=\frac{d \tau-b}{-\Delta \tau+2}$, we obtain

$$
\varphi_{\delta, \rho, 3}(\tau)=\sum_{k=0}^{\infty} a_{k}^{\prime} \exp \left\{2 \pi i k S^{-1} \tau /(2 n / \Delta)\right\}
$$

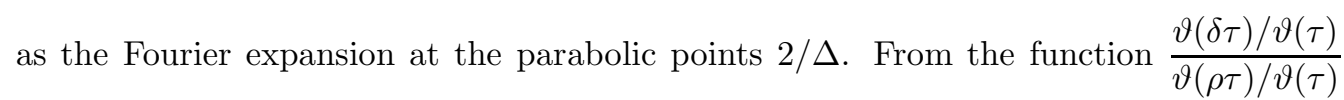
and the equation

$\vartheta(\delta S \tau) / \vartheta(S \tau)=c^{\prime} \prod_{m=1}^{\infty}\left(1-c^{2 m} q^{2 m \Delta k / n}\right)\left(1+c^{2 m-1} q^{(2 m-1) \Delta k / n}\right)^{2}\left(1-q^{2 m}\right)^{-1}\left(1+q^{2 m-1}\right)^{-2}$

in [7, Theorem 4], it follows that the valence $\nu$ is 0 . Where $k=n g / \Delta \delta_{0}, q=\exp (i \pi \tau)$ and $c, c^{\prime}$ are non-zero constants.

It is shown in [7] that $f(N \tau)$ has the form

$$
f(N \tau)=\sum_{k=0}^{\infty} b_{k} \exp \{2 \pi i(k+\nu) \tau /(n / \Delta)\}
$$

at the parabolic points $1 / \Delta$. Hence, we have

$$
\varphi_{\delta, \rho, 3}(N \tau)=\sum_{k=0}^{\infty} b_{k}^{\prime} \exp \{2 \pi i(k+\nu) \tau /(n / \Delta)\}
$$

where the $b_{k}^{\prime}$ are complex numbers with $b_{0} \neq 0$ and $N=\left(\begin{array}{cc}1 & 0 \\ \Delta & 1\end{array}\right)$. Hence, replacing $\tau$ by $N^{-1} \tau=\frac{\tau}{-\Delta \tau+1}$, we obtain

$$
\varphi_{\delta, \rho, 3}(\tau)=\sum_{k=0}^{\infty} b_{k}^{\prime} \exp \left\{2 \pi i(k+\nu) N^{-1} \tau /(n / \Delta)\right\}
$$

as the Fourier expansion at the parabolic points $1 / \Delta$. From the function $\frac{\vartheta(\delta \tau) / \vartheta(\tau)}{\vartheta(\rho \tau) / \vartheta(\tau)}$ and the equation

$\vartheta(\delta N \tau) / \vartheta(N \tau)=c^{\prime \prime} z^{(k-n / \Delta) / 8} \prod_{m=1}^{\infty}\left(1-c^{2 m} z^{2 m k}\right)\left(1+c^{m} z^{m k}\right)\left(1-z^{2 m n / \Delta}\right)^{-1}\left(1+z^{m n / \Delta}\right)^{-1}$ 
in $[7$, Theorem 5], it is clear that the valence $\nu$ is

$$
\frac{1}{8} \sum_{\delta|n, \rho| n}\left\{\left(n g / \Delta \delta_{0}\right)-\left(n g^{\prime} / \Delta \rho_{0}\right)\right\} r_{\delta}
$$

where $g=(\delta, \Delta), \delta_{0} g=\delta, \rho_{0} g^{\prime}=\rho, g^{\prime}=(\rho, \Delta), z=\exp (2 \pi i \Delta \tau / n), k=n g / \Delta \delta_{0}$, $c=\exp \left(-2 \pi i \beta / \delta_{0}\right), c^{\prime \prime}=c^{\prime} \exp \left(-\pi i \beta / 4 \delta_{0}\right)$.

Theorem 2. Let $n, \delta, \rho, r_{\delta}$ be as in Theorem 1. If the conditions (5) hold, then $\varphi_{\delta, \rho, i}(\tau)$ are modular functions on $P^{-2} \theta(n) P^{2}$ and $P^{-1} \theta(n) P$, for $i=2,4$, respectively.

Proof. By Lemma 2, using the equality $\vartheta_{3}(\tau) \mid P=e^{-\frac{1}{4} \pi i} \vartheta_{4}(\tau)$, we have

$$
\varphi_{\delta, \rho, 3}(\tau) \mid P=\varphi_{\delta, \rho, 4}(\tau)
$$

By Lemma 1, $\varphi_{\delta, \rho, 4}(\tau)$ is a modular function on $P^{-1} \theta(n) P$. By Lemma 2, using the equality $\vartheta_{3}(\tau) \mid P^{2}=e^{-\frac{1}{4} \pi i} \vartheta_{2}(\tau)$, we obtain

$$
\varphi_{\delta, \rho, 3}(\tau) \mid P^{2}=\varphi_{\delta, \rho, 2}(\tau)
$$

By Lemma $1, \varphi_{\delta, \rho, 2}(\tau)$ is a modular function on $P^{-2} \theta(n) P^{2}$. This concludes the proof.

\section{The Modular Functions $T_{m, n, i}(\tau), H_{m, n, i}(\tau), \Phi_{m, n, i}(\tau)$}

Now we consider the following functions:

$T_{m, n, i}(\tau)=\frac{\vartheta_{i}(\tau) \vartheta_{i}(n \tau)}{\vartheta_{i}(m \tau) \vartheta_{i}(m n \tau)}, \quad H_{m, n, i}(\tau)=\frac{\vartheta_{i}(m \tau) \vartheta_{i}(n \tau)}{\vartheta_{i}(\tau) \vartheta_{i}(m n \tau)}$, and $\Phi_{m, n, i}(\tau)=\frac{\vartheta_{i}(m \tau)}{\vartheta_{i}(n \tau)}$, for $i=2,3,4$

The following theorems show that, under appropriate conditions $T_{m, n, i}^{r}(\tau), H_{m, n, i}^{r}(\tau)$ and $\Phi_{m, n, i}^{r}(\tau)$ are modular functions holomorphic on $\chi$, with respect to the transformations of appropriate subgroups of finite index of the modular group, i.e., modular forms of weight 0 .

Theorem 3. Let $m$ and $n$ denote positive integers and suppose that $r$ is an integer such that $r(m-1)(n+1) \equiv 0(\bmod 8)$. Then $T_{m, n, 3}^{r}(\tau) \in M(\theta(m n), 0,1)$. Moreover, $T_{m, n, 3}^{r}(\tau)$ is analytic on $\chi$.

Proof. The last assertion in Theorem 3 is obvious from the definition of $T_{m, n, 3}(\tau)$. Let $A=\left(\begin{array}{ll}a & b \\ c & d\end{array}\right) \in \theta(m n)$. Then, for $\tau \in \chi$,

$$
\vartheta(A \tau)=\nu(A)(c \tau+d)^{1 / 2} \vartheta(\tau)
$$

and for $s \mid c$,

$$
\vartheta(s A \tau)=\vartheta\left(\frac{a(s \tau)+s b}{\frac{c}{s}(s \tau)+d}\right)=\nu\left(\begin{array}{lr}
a & s b \\
c / s & d
\end{array}\right)(c \tau+d)^{1 / 2} \vartheta(s \tau)
$$


where $\nu(A)$ is given by (4). Thus,

$$
T_{m, n}(A \tau)=\nu_{1}(A) T_{m, n}(\tau)
$$

where $\nu_{1}(A)=\frac{\nu\left(\begin{array}{cc}a & b \\ c & d\end{array}\right) \nu\left(\begin{array}{cc}a & n b \\ c / n & d\end{array}\right)}{\nu\left(\begin{array}{cc}a \\ c / m b\end{array}\right) \nu\left(\begin{array}{cc}a & m b b \\ c / m n & m b\end{array}\right)}$. Suppose first that $A \equiv V(\bmod 2)$. Then from (4),

$$
\nu_{1}(A)=\zeta_{8}^{-\left(c+\frac{c}{n}-\frac{c}{m}-\frac{c}{m n}\right)}=\zeta_{8}^{-c(m-1)(n+1) / m n}
$$

Since $r(m-1)(n+1) \equiv 0(\bmod 8), \nu_{l}^{r}(A) \equiv 1$. Where $\zeta_{8}=\exp (2 \pi i / 8)$.

Secondly, suppose that $A \equiv I(\bmod 2)$. Then $\nu_{l}^{r}(A) \equiv 1$. Thus, in both instances,

$$
T_{m, n, 3}^{r}(A \tau)=T_{m, n, 3}^{r}(\tau)
$$

For $\delta=1, \rho=m$ and $\delta=n, \rho=m n$ in the equation (8), we have the Fourier expansion of $T_{m, n, 3}^{r}(\tau)$ at 0 of the form

$$
T_{m, n, 3}^{r}(\tau)=m^{r} . \exp \frac{\pi i r}{8 \tau}\left(\frac{1-m}{m}+\frac{n-m n}{m n^{2}}\right)\left(1+\sum_{k=1}^{\infty} \alpha_{k} e^{-\frac{2 \pi i k}{\tau} \gamma(m, n)}\right)
$$

where, $\gamma(m, n)$ is a rational function of $m$ and $n$. Similarly, from the equations (7), (9), (11), we obtain the Fourier expansions of $T_{m, n, 3}^{r}(\tau)$ at the indicated other cusp points of the forms

$$
\begin{aligned}
& T_{m, n, 3}^{r}(\tau)=1+\sum_{k=1}^{\infty} a_{k}^{\prime} e^{2 \pi i k \tau}, \quad \text { at } \infty \\
& T_{m, n, 3}^{r}(\tau)=\sum_{k=0}^{\infty} a_{k}^{\prime} \exp \left\{2 \pi i k S^{-1} \tau /(2 m n / \Delta)\right\}, \quad \text { at } 2 / \Delta \\
& T_{m, n, 3}^{r}(\tau)=\sum_{k=0}^{\infty} b_{k}^{\prime} \exp \left\{2 \pi i(k+\nu) N^{-1} \tau /(m n / \Delta)\right\}, \quad \text { at } 1 / \Delta,
\end{aligned}
$$

From the function $\frac{\vartheta(n \tau) / \vartheta(\tau)}{(\vartheta(m \tau) / \vartheta(\tau))(\vartheta(m n \tau) / \vartheta(\tau))}$ and the equation (10), we find that $T_{m, n, 3}^{r}(\tau)$ has valence 0 at the parabolic points $2 / \Delta$. From the same function and the equation (12), $T_{m, n, 3}^{r}(\tau)$ has valence

$$
\frac{1}{8}\left\{(m n / \Delta)+\left(m n g^{\prime} / \Delta n_{0}\right)-\left(m n g^{\prime \prime} / \Delta m_{0}\right)-\left(m n g^{\prime \prime \prime} / \Delta m_{0} n_{0}\right)\right\} r
$$

at the parabolic points $1 / \Delta(\Delta \mid m n, \Delta>0)$, where $g^{\prime}=(n, \Delta), g^{\prime \prime}=(m, \Delta), g^{\prime \prime \prime}=$ $(m n, \Delta), n_{0} g^{\prime}=n, m_{0} g^{\prime \prime}=m, m_{0} n_{0} g^{\prime \prime \prime}=m n$.

Theorem 4. Let $m, n, r$ be as in Theorem 3. Then, $T_{m, n, 4}^{r}(\tau) \in M\left(P^{-1} \theta(m n) P, 0,1\right)$ and $T_{m, n, 2}^{r}(\tau) \in M\left(P^{-2} \theta(m n) P^{2}, 0,1\right)$ 
Proof. Using the equations (2), we have $T_{m, n, 3}^{r}(\tau) \mid P=T_{m, n, 4}^{r}(\tau)$ and $T_{m, n, 3}^{r}(\tau) \mid$ $P^{2}=T_{m, n, 2}^{r}(\tau)$. Hence, we have the conclusion.

Theorem 5. Let $m$ and $n$ denote positive integers and suppose that $r$ is an integer such that $r(m-1)(n-1) \equiv 0(\bmod 8)$. Then, $H_{m, n, 3}^{r}(\tau) \in M(\theta(m n), 0,1)$. Moreover, $H_{m, n, 3}^{r}(\tau)$ is analytic on $\chi$.

Proof. The proof is analogous to that of Theorme 3.

Theorem 6. Let $m, n, r$ be as in Theorem 5. Then, $H_{m, n, 4}^{r}(\tau) \in M\left(P^{-1} \theta(m n) P, 0,1\right)$ and $H_{m, n, 2}^{r}(\tau) \in M\left(P^{-2} \theta(m n) P^{2}, 0,1\right)$.

Proof. As the proof of Theorem 4, from the equations $H_{m, n, 3}^{r}(\tau) \mid P=H_{m, n, 4}^{r}(\tau)$ and $H_{m, n, 3}^{r}(\tau) \mid P^{2}=H_{m, n, 2}^{r}(\tau)$, the assertion follows.

Theorem 7. Let $m$ and $n$ denote positive integers and suppose that $r$ is an integer such that $r^{2}(n-m)(n m-1) \equiv 0(\bmod 8)$. Then, $\Phi_{m, n, 3}^{r}(\tau)$ is a modular function on $\theta(m n)$, with multiplier system $\left(\frac{d}{m n}\right)^{r}$.

Proof. Let $A=\left(\begin{array}{ll}a & b \\ c & d\end{array}\right) \in \theta(m n)$. Then for $\tau \in \chi$

$$
\Phi_{m, n, 3}(A \tau)=\frac{\vartheta(m A \tau)}{\vartheta(n A \tau)}=\frac{\vartheta\left(A_{1} m \tau\right)}{\vartheta\left(A_{2} n \tau\right)}=\frac{\nu\left(A_{1}\right)}{\nu\left(A_{2}\right)} \frac{\vartheta(m \tau)}{\vartheta(n \tau)}
$$

where $A_{1}=\left(\begin{array}{cc}a & m b \\ c / m & d\end{array}\right), A_{2}=\left(\begin{array}{cc}a & n b \\ c / n & d\end{array}\right)$. If $A_{1}, A_{2} \equiv V(\bmod 2)$, by (4), $\nu(A)=\left(\frac{d}{m n}\right) e^{-\frac{\pi i c}{4}\left(\frac{n-m}{n m}\right)}$. Since $r(n-m) \equiv 0(\bmod 8)$, we have $\nu^{r}(A)=\left(\frac{d}{m n}\right)^{r}$. If $A_{1}, A_{2} \equiv I(\bmod 2)$, by $(4)$ and quadratic reciprocity law, we have $\nu(A)=\left(\frac{n m}{d}\right)=$ $\left(\frac{d}{n m}\right)(-1)^{\left(\frac{n m-1}{2}\right)\left(\frac{d-1}{2}\right)}$. Since $r(n m-1) \equiv 0(\bmod 8), \nu^{r}(A)=\left(\frac{d}{n m}\right)^{r}$. Thus, in both instances, we have $\Phi_{m, n, 3}^{r}(A \tau)=\nu^{r}(A) \Phi_{m, n, 3}^{r}(\tau)$. Now we consider the Fourier epansions of $\Phi_{m, n, 3}^{r}(\tau)$ at the cusps of $\theta(m n)$. For $\delta=m, \rho=n$ in the equations (7), (8), (9), (11), we obtain the Fourier expansions of $\Phi_{m, n, 3}^{r}(\tau)$ at the parabolic points $\infty, 0,2 / \Delta, 1 / \Delta$, respectively, $(\Delta \mid m n, \Delta>0)$. From the function $\frac{\vartheta(m \tau) / \vartheta(\tau)}{\vartheta(n \tau) / \vartheta(\tau)}$ and the equation (10), we note that $\Phi_{m, n, 3}^{r}(\tau)$ has valence 0 at the parabolic points $2 / \Delta$. From the same function and the equation (12), we find that $\Phi_{m, n, 3}^{r}(\tau)$ has valence

$$
\frac{1}{8}\left\{\left(m n g / \Delta m_{0}\right)-\left(m n g^{\prime} / \Delta n_{0}\right)\right\} r
$$

at the parabolic point $1 / \Delta$, where $g=(m, \Delta), m_{0} g=m, g^{\prime}=(n, \Delta), n_{0} g^{\prime}=n$. Thus $\Phi_{m, n, 3}^{r}(\tau) \in M\left(\theta(m n), 0,\left(\frac{d}{m n}\right)^{r}\right)$. 
Theorem 8. Let $m, n, r$ be as in Theorem 7. Then $\Phi_{m, n, 4}^{r}(\tau) \in M\left(P^{-1} \theta(m n) P, 0,1\right)$ and $\Phi_{m, n, 2}^{r}(\tau) \in M\left(P^{-2} \theta(m n) P^{2}, 0,1\right)$.

Proof. By the equations (2), since $\Phi_{m, n, 3}^{r}(\tau) \mid P=\Phi_{m, n, 4}^{r}(\tau)$ and $\Phi_{m, n, 3}^{r}(\tau) \mid P^{2}=$ $\Phi_{m, n, 2}^{r}(\tau)$ the assertion follows.

\section{Conclusion}

It is a simple matter, using the work already done in this paper, to formulate and prove analogous results for functions in $\Gamma_{0}(2 m n)$. The key is the equation $\Gamma_{0}(2 m n)=$ $W^{-m n} \theta(m n) W^{m n}$, where $m$ and $n$ are odd positive integers. The functions $T_{m, n, 3}\left(W^{m n} \tau\right)$, $H_{m, n, 3}\left(W^{m n} \tau\right)$ and $\Phi_{m, n, 3}\left(W^{m n} \tau\right)$ are modular functions on $\Gamma_{0}(2 m n)$. The functions $\varphi_{\delta, \rho, 3}\left(W^{n} \tau\right)$ are modular functions on $\Gamma_{0}(2 n)$, for an odd square-free positive integer $n$. For example, the functions $T_{m, n, 3}\left(W^{m n} \tau\right)$ are modular functions on $\Gamma_{0}(2 m n)$ with valence 0 at the parabolic points $W^{-m n} S(i \infty)$ and valence (13) at the parabolic points $W^{-m n+\Delta}(i \infty),(\Delta \mid m n, \quad \Delta>0)$, where $S=\left(\begin{array}{cc}2 & b \\ \Delta & d\end{array}\right)$ and $W=\left(\begin{array}{ll}1 & 0 \\ 1 & 1\end{array}\right)$. In this setting, the natural parabolic point in which to expand the function is $i \infty$.

\section{References}

[1] Berndt, B. C., Cheng Zhang-Liang, Ramanujan's identities for eta functions. Math. Ann. 292(1992), 561-573.

[2] Kirmaci, U. S., Özdemir, M. E., On the modular functions, International Journal of Applied Mathematics, 2(2000), 1385-1397.

[3] Knopp, M. I., Modular Functions in Analytic Number Theory, Markham Publishing Co, Chicago, 1970.

[4] Newman, M., Construction and application of a class of modular functions, Proc. London Math. Soc. 7(1957), 334-350.

[5] Newman, M., Construction and application of a class of modular functions II, Proc. London Math. Soc. 9(1959), 373-387.

[6] Rankin, R., Modular Forms and Functions, Cambridge University Press, Cambridge, 1977.

[7] Wilson, G., A family of modular functions arising from the theta function, Proc. London Math. Soc. 55(1987), 433-449.

Atatürk University, K. K. Education Faculty, Department of Mathematics, 25240, ErzurumTurkey. 